\title{
Structure Optimization Design of 4-UPU Parallel Mechanism
}

\author{
Xingwei Hu \\ College of mechanical engineering \\ Jiamusi University \\ Jiamusi154007 Heilongjiang China \\ 975658280@qq.com
}

\section{Guanglan Xia}

College of mechanical engineering

Jiamusi University

Jiamusi154007 Heilongjiang China

xiaguanglan@163.com

Feng $\mathrm{Yu}$

College of mechanical engineering Jiamusi University

Jiamusi154007 Heilongjiang China

yufenglaoshi@163.com

\author{
Baolin Yin \\ College of mechanical engineering \\ Jiamusi University \\ Jiamusi154007 Heilongjiang China \\ yinbaolin@163.com \\ Haijuan Ding \\ College of mechanical engineering \\ Jiamusi University \\ Jiamusi154007 Heilongjiang China \\ dinghaijuan@163.com \\ Yangyao Liu \\ College of mechanical engineering \\ Jiamusi University \\ Jiamusi154007 Heilongjiang China \\ liuyangyao@163.com
}

\begin{abstract}
UPU belongs to the category of less degree of freedom parallel mechanism, this paper analyzes kinematic pair of the parallel mechanism and the structure design of key parts, and selecting the best design scheme.According to the bottom and the principle finish three-dimensional entity assembly, in the end we can get the frame structure of 4-UPU parallel mechanism.
\end{abstract}

Keywords-optimization design; the key components; from the bottom up;ontology structure; parallel mechanism

\section{INTRODUCTION}

At the beginning of the 21st century before and after 10 years, the international institutions conducted to learn the research of less degrees of freedom parallel mechanism. Less degrees of freedom parallel mechanism can be defined as:It uses $2 \sim 5$ movement branched chain to link between moving platform and the static platform , in general it has $2 \sim 5$ degrees of freedom, and selecting a drive device on every movement the branched chain, so this mechanism is called less degrees of freedom parallel mechanism. Like in this paper, the 4-UPU parallel mechanism has four branched chain, and each movement of the branched chain structure is symmetry, which institution has four independent degrees of freedom, so it can realize 3T1Rz movement.

Because the agency is composed of artifacts and kinematic pair generally, and they constitute the basic elements of space agencies. The complexity of the space agency is its many motion pair of distribution in space. The study of 4-UPU structure also started from motion pair, and then selecting the best design optimization.
So far, the development of less degrees of freedom parallel mechanism model is relatively less, and like Delta institutions, 3-RPS institutions, 3-UPU institutions, etc. In this paper, the development of 4-UPU parallel mechanism was put forward by professor Huang zhen of Yanshan university, so the understanding and design of its structure have a certain practical significance.

\section{MOTION PAIR OF PARALLEL MECHANISM}

Motion pair of parallel mechanism has revolute pair, prismatic pair, cylindrical vice, spherical pair, universal joint and so on.

(1)revolute pair: It allows two components to finish rotation axis relatively, and with a relative degree of freedom, $\mathrm{F}=1$.

(2)prismatic pair: It allows two components to finish movement along the axis relatively, and with a relative degree of freedom, $\mathrm{F}=1$.

(3) universal joint: It allows two components to have two relative rotational movement, and with two degrees of freedom, $F=2$, universal joint is equal to the axis of the intersection of two rotation $(\mathrm{U}=\mathrm{RR})$.

\section{4-UPU PARALLEL MECHANISM DESIGN OF KEY PARTS}

The design of $U$ vice I regard universal joint for reference in this article, and it realizes relative rotation in two axes of rotation of the intersection by two step shafts and bearing. $U$ vice I compose of two rotations which is the $R$ vice I and $R$ vice II, the axis of $R$ vice I parallel to $R$ vice I of $U$ vice II, and parallel to the $\mathrm{Z}$ axis; the axis of $\mathrm{R}$ 
vice II parallel to $R$ vice II of $U$ vice II each other and perpendicular to the $\mathrm{Z}$ axis, it is shown in Figure 1 . The scheme one can meet 4- UPU the movement requirements, through static plate flange mounted on the prototype of the location to guarantee the precision of fabrication and installation process, and as a result of the milling surface is relatively narrow, so it is put forward higher requirements for the milling process, and it is not convenient to process. In view of this, amending the scheme into Figure 2, and getting a relatively large round face milling, it not only ensure the machining accuracy, and relatively reasonable for machining process.

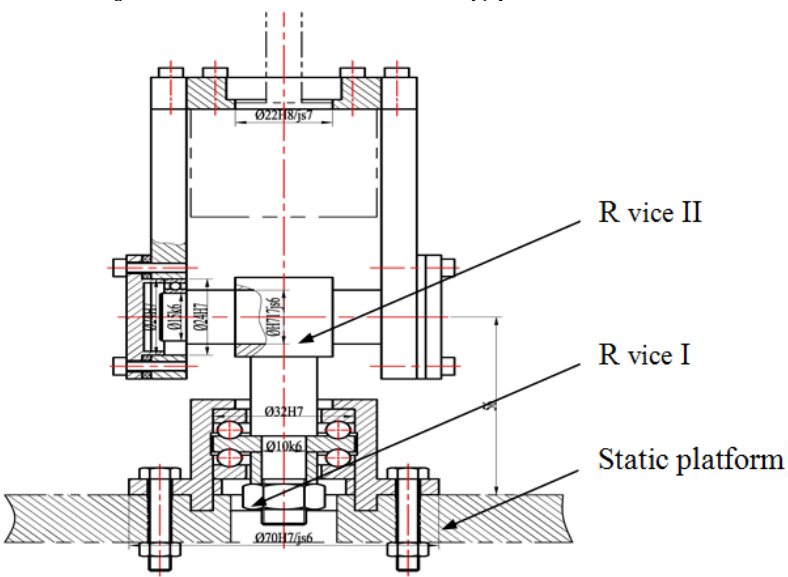

Figure 1. Scheme one U vice I

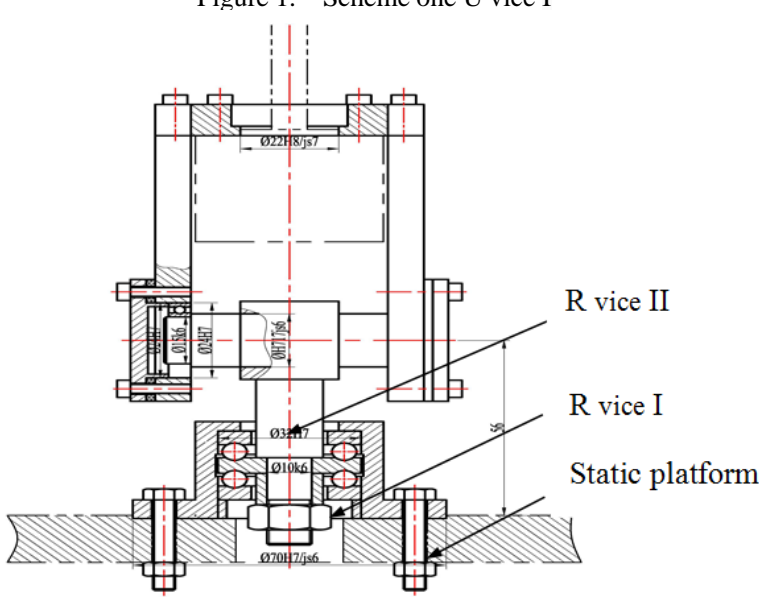

Figure 2. Scheme two U vice I

$\mathrm{P}$ pair regard motor as power source, it realize movement up and down through the screw motor drive board 1 and mobile plate 2 along the axis direction. Sliding bearing choose flange linear sliding bearing, it can ensure the accuracy of positioning and installation is convenient, and can guarantee the trajectory. It is shown in Figure 3 Scheme one it place a protrusion of in fixed plate 2 which the polished rod hollowed out, this design can guarantee good stiffness, but it not ensure the accuracy of motion well, as well as fixed bigger challenges are put forward that is from mobile plate 1 out of the polished rod. From Figure 4 scheme two, it fixed installation of bearing plate on the whole the scheme one, this design makes trajectory accurately, and then it also put forward higher requirements for positioning and design. So amending the design of scheme three in Figure 5, this scheme will fix polished rod on both sides for mobile plate 1 , this design makes the fixed position is relatively simple, but the force on both sides make the stiffness decrease, then using two identical mobile plate increase the stiffness of the agency. It make the moving track more accurate, and weakened the design defects, at the same time to enhance the operational and practical.
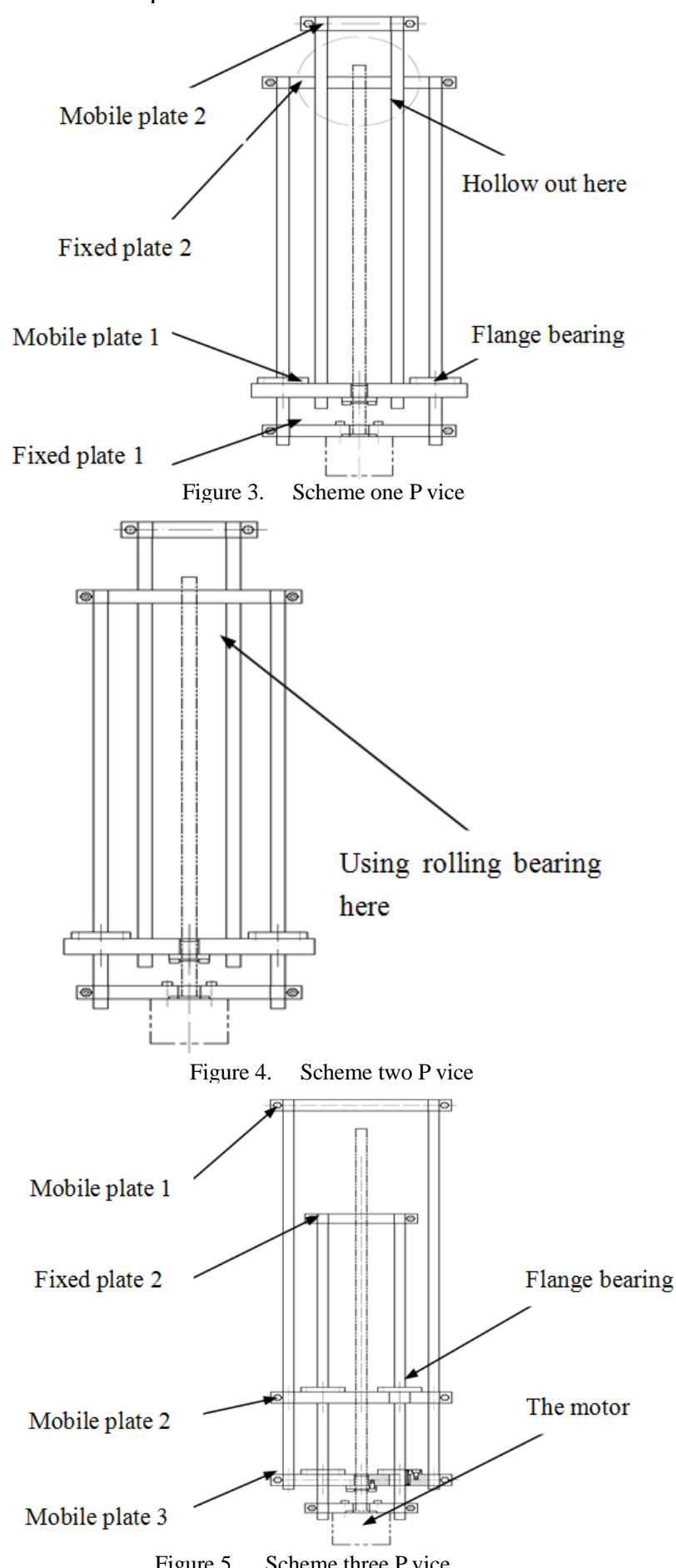

The design principle of $U$ vice II is the same with $U$ vice I, and three schemes are given in Figure $6 \sim$ Figure 8 , in the end we choose the best design scheme three. 


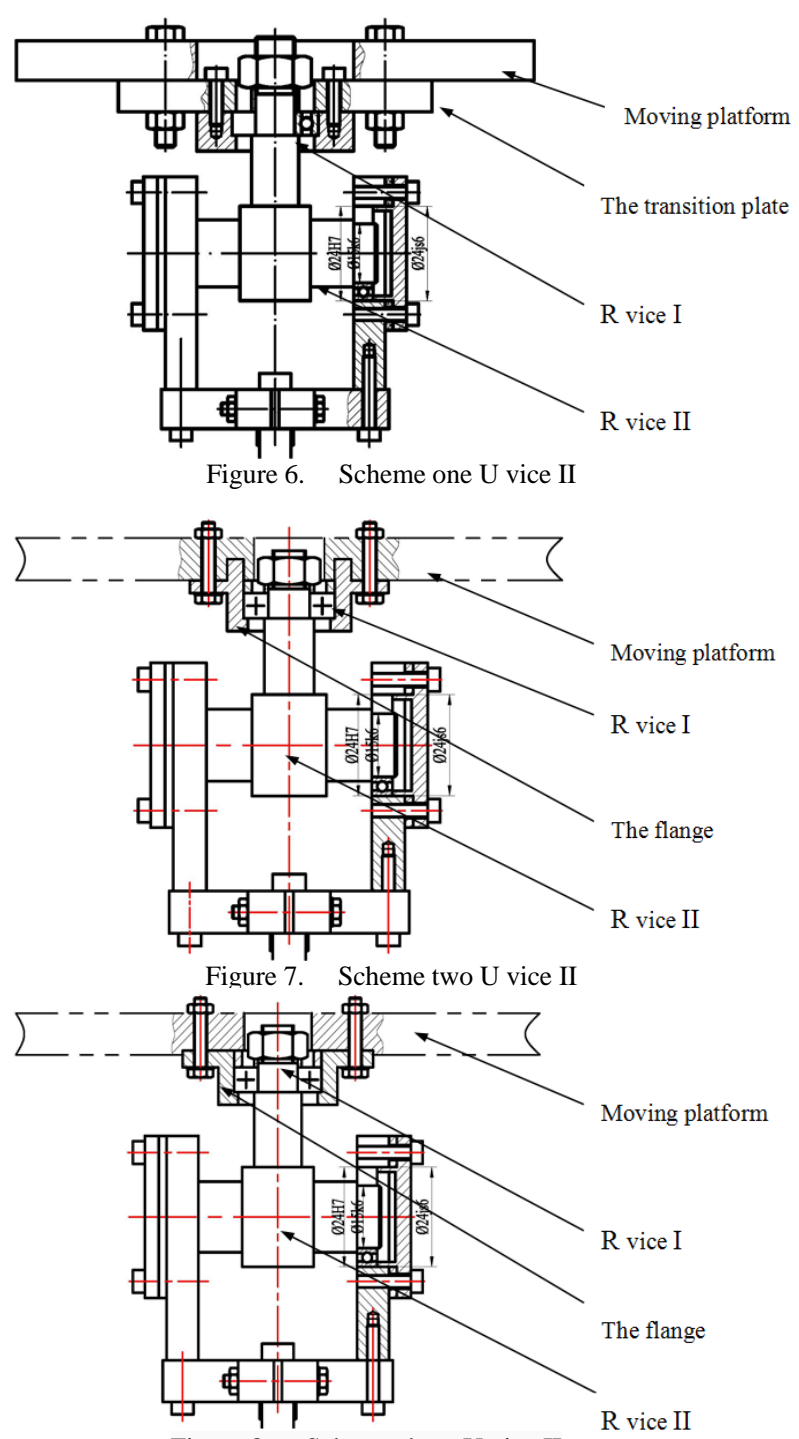

Figure 8. Scheme three U vice II

As shown in Figure 9 which is 4-UPU parallel mechanism assembly drawing, this mechanism regard four identical and symmetrical distribution of motor as power source. Moving platform and static platform connected to with $\mathrm{U}$ vice I, P vice, U vice II. And it achieve $3 T 1 R^{z}$. As shown in figure from 10 to 13 , it respectively showed 4-UPU parallel mechanism along the $\mathrm{x}, \mathrm{y}, \mathrm{z}$ axis direction of move and turn the situation around the $\mathrm{z}$ axis.

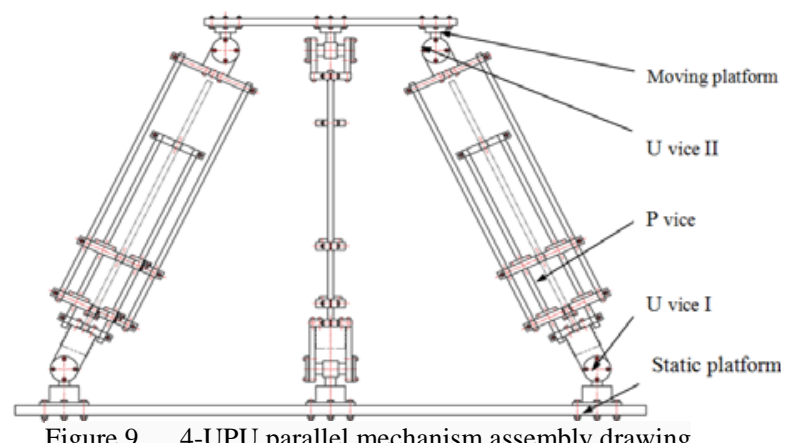

Figure 9. 4-UPU parallel mechanism assembly drawing
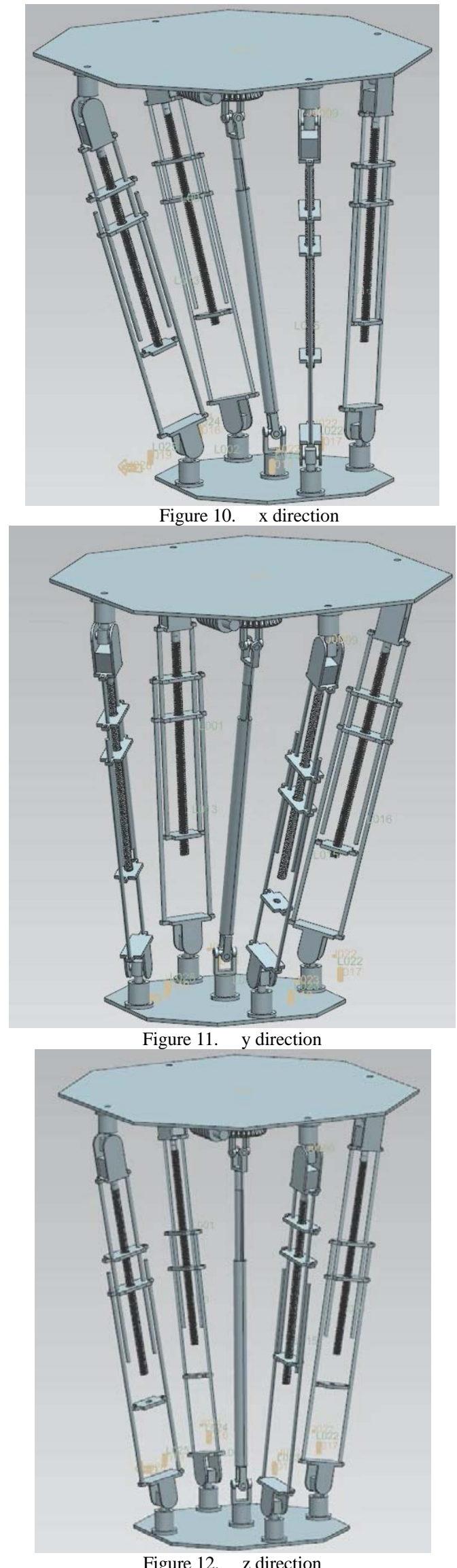

Figure 12. $\mathrm{z}$ direction 


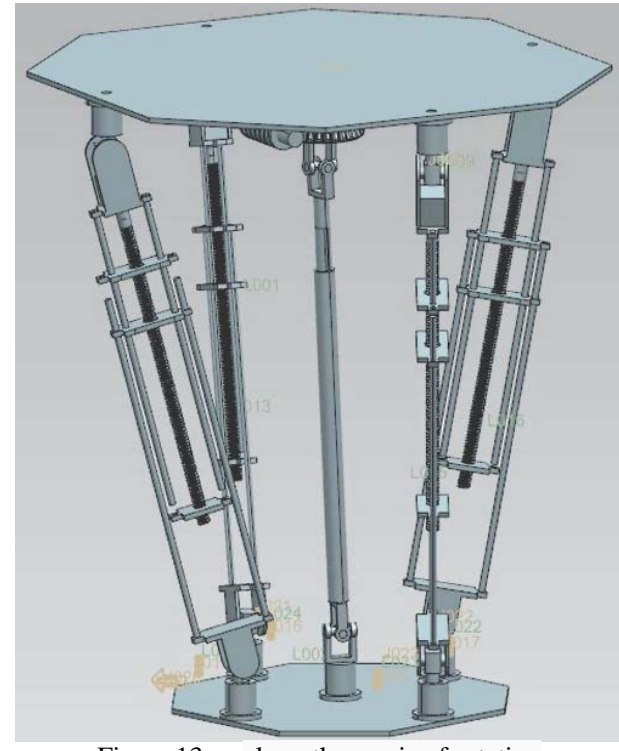

Figure 13. along the $\mathrm{z}$ axis of rotation

\section{CONCLUSION}

This paper mainly analyze parts design which aims at $U$ vice $I, P$ vice, $U$ vice II. First of all, the basic components of 4-UPU institutions are designed based on agency theory; Secondly, It finally chooses the optimal structure design by a variety of design schemes comparison and analysis according to the cooperate relationship between parts and components and positioning in the process of machining requirements; Finally, 4-UPU ontology structure makes possible by processing requirements and positioning relationship.

\section{ACKNOWLEDGMENT}

This work was financially supported by Heilongjiang Department of Education(12531672), Science and Technology Project of the Jiamusi University in
Heilongjiang province(Lz2014-003), Science and Technology Innovation Project of postgraduate of Jiamusi University in Heilongjiang Province(LM2014-008). In addition to them, it was supported by Jiamusi University Graduate student academic exchange program.

THE CORRESPONDING AUTHOR: Guanglan Xia

\section{REFERENCES}

[1] Zhen Huang,Yongsheng Zhao, Tieshi Zhao. Advanced spatial mechanism [M].Beijing: Higher Education Press, 2006.

[2] Zhao Tie-shi,Wang Guchun, etc. 4-UPU parallel robot mechanism and its kinematics [J]. China mechanical engineering, 2005 (22).

[3] D.Z latanov, c. Gosselin. A New Parallel Architecture with Four Degrees of Freedom [C]. The 2nd workshop on Computational Kinematics, Seoul, 2001:57-66.

[4] Jianmin Liu, Lvzhong Ma. Spatial four degree of freedom parallel robot intelligent type comprehensive [J]. Mechanical design and manufacture, 2008 (6): 160-162.

[5] L H. Rolland. The manta and the kanuk novel 4-DOF parallel mechanisms for industrial Handling[C].In: Proc ASME Int Conf on Mechanical Engineering. Nashville: TN, 1999: 253-258.

[6] Haibing Feng. 4-HUPU less degree of freedom parallel mechanism kinematics modeling and analysis of J. Modern manufacturing engineering, 2013 (4): 30-34.

[7] Dachang Zhu, Wenjun Feng,Yunhong Liu. Influence coefficient 3RPS parallel mechanism, kinematics analysis and Simulation of $J$ based on. Mechanical transmission, 2014 (3): 76-78.

[8] Jinfeng Wang, Xiaoliang Fan. Study on the development of a J parallel robot. International Conference, 2010 (12).

[9] Yi Lu, Bo Hu. Research progress of less degree of freedom parallel mechanism J. Journal of Yanshan University, 2011 (9).

[10] J. Lenarcic, M. M. Stanisic, V. Parenti-Castelli. A 4-DOF parallel mechanism simulating the movement of the human sternum clavicle scapula complex[M]. In: Advances in Robot Kinematics.London: Kluwer Academic Publishers, 2000: 325-332.

[11] Fujiu Yang, Guangqi Cai, Jiman Luo, et al. The less degree of freedom parallel robot research status of $\mathrm{J}$ machine tool \& hydraulics, 